\title{
The Never Ending Debate Single-Layer Versus Double-Layer Closure of the Uterine Incision at Cesarean Section
}

\author{
Hegde C. V.
}

Received: 17 April 2014/Accepted: 14 May 2014/Published online: 23 July 2014

(C) Federation of Obstetric \& Gynecological Societies of India 2014

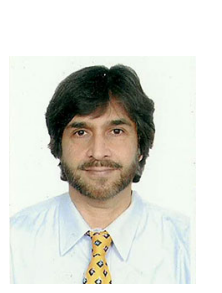

\begin{abstract}
About the Author
C. V. Hegde is a professor and chief of unit at the T N Medical and B Y L Nair Hospital, Mumbai, India. He is an endoscopic surgeon and has a deep interest in performing surgery to correct pelvic floor defects. His surgical videos are accessible on YouTube and are meant for teaching purposes. He has performed endoscopic and advanced vaginal surgery at many workshops. He is a passionate teacher who would long to go to places to give a good talk. He has been post graduate and undergraduate teacher, Bombay University and Maharashtra University of Health Sciences for twenty five years. He has several publications to his credit and has been an invited speaker at national and international conferences.
\end{abstract}

In the beginning-a caveat. This short review article will not discuss all aspects of cesarean sections but will be finely nuanced and limit itself to the merits/demerits of a single-layer closure of the uterine incision versus a doublelayer closure. Therefore, references have been chosen which are relevant, recent, and which bring some value addition to the discussion. This is also not the place to discuss any particular "new" method of uterine incision closure unless it has been exhaustively practiced and reviewed after its introduction.

All through the twentieth century barring exceptions, the uterus was probably sutured in two layers at cesarean section. The sutures were chromic catgut and as described in Munro Kerr, the first layer was probably a continuous

Hegde C. V. ( $₫)$, Professor and Chief

Department of Obstetrics and Gynecology, T N Medical College

and B Y L Nair Hospital, Mumbai Central,

Mumbai 400 008, India

e-mail: dr.c.v.hegde@gmail.com locking one and the second layer was continuous and meant to "bury" the first. A few hardy souls I suspect must have dared even then to suture the uterus in one layer with chromic catgut.

Now that suturing is done with delayed synthetic absorbable material like polyglactin, which has among its properties a greater tensile strength for a longer period of time and delayed degradation as compared to chromic catgut, suturing the uterine incision at cesarean section in a single layer seems empirically to have more takers. Then, there are those who have traditionally sutured the uterus at cesarean section in two layers and are loathe to change a practice which has stood them in good stead.

The questions raised by any method departing from a set template include those of safety, efficacy, and complications both immediate and delayed. The proponents of the single-layer closure with polyglactin often cite advantages of this method being efficient, quick, safe, less cumbersome, and reduced bleeding as compared to the two-layered closure. It would stand to reason that whatever the 
methodology of closure the twin objectives that must be met are safe hemostasis and an insurance against rupture uterus in a future trial of labor.

In a multicenter case control study [1], where the objective was "to evaluate the effects of prior single-layer compared with double-layer closure on the risk of uterine rupture," the cases chosen were those who had a previous single transverse lower segment incision and had a complete rupture of the uterus during a trial of labor. The controls (288) were three times the cases (96) and comprised women who had a prior low transverse uterine incision and underwent trial of labor without rupture. Multiple variables included risk factors such as "prior uterine closure, suture material, diabetes, prior vaginal delivery, labor induction, cervical ripening, birth weight, prostaglandin use, maternal age, gestational age, and interdelivery interval." The rate of single-layer closure was $36 \%$ (35 of 96) in the case group and $20 \%$ (58 of 288$)$ in the control group $(P<0.01)$. Singlelayer closure was related to uterine rupture associated with adverse neonatal outcome [odds ratio (OR) 2.89; $95 \%$ confidence interval (CI) 1.01-8.27]. Single-layer closure (OR 2.69; $95 \%$ CI 1.37-5.28) and birth weight greater than 3,500 g (OR 2.03; $95 \%$ CI 1.21-3.38) were also linked with increased rates of uterine rupture. The authors concluded that "a prior single layer closure carries more than twice the risk of uterine rupture compared with a double layer closure and that single-layer closure should be avoided in women who could contemplate future vaginal birth after cesarean delivery." The study had a level II evidence.

A review article in the International Journal of Gynecology and Obstetrics [2] evaluated "the best available evidence regarding the association between single-layer closure and uterine rupture." The methodology included a Medline, Embase, and Cochrane database search for "relevant observational and experimental studies that included women with a previous single, low, transverse cesarean delivery who had attempted a trial of labor (TOL)." 5,810 women were reviewed in nine studies and "a sensitivity analysis indicated that the risk of uterine rupture was increased after a locked single-layer closure (OR 4.96; $95 \%$ CI 2.58-9.52, $P<0.001)$ but not after an unlocked single-layer closure (OR 0.49; $95 \%$ CI 0.21-1.16), compared with a double-layer closure." The study concludes that a locked single layer rather than unlocked single layer was associated with a higher risk of rupture than a double layer in women after trial of labor.

A detailed Cochrane database review [3] reviewed several variables including uterine closure at cesarean section. The search method included the Cochrane Pregnancy and Childbirth Group's Trials Register (Nov 2007). This search was updated on May 31, 2012. The selection criteria included "all published, unpublished, and ongoing randomised control led trials comparing various types and closure of uterine incisions during Cesarean section." The authors came to the conclusion that there was no information regarding either the optimal suture material or a suturing technique for the uterine incision. In an article [4], "subjects with one previous cesarean section by documented transverse uterine incision that attempted VBAC were identified. Uterine rupture and VBAC success rates were compared between those with single-layer and double-layer uterine closure. Of 948 subjects identified, 913 had double-layer closure and 35 had single-layer closure. The uterine rupture rate was significantly higher in the single-layer closure group (8.6 vs. $1.3 \%, P=0.015$ )." In another study, where the merits and demerits of a single-layer closure of the uterine incision in a continuous and continuous locked manner were compared by Hudic et al. [5] and there was no significant difference in uterine scar disruption between the two groups. This was by means of a retrospective cohort study in a population, where unlocked and locked sutures were used. This study further nuances a single-layer closure method but contradicts a finding in a study mentioned earlier in this article. The authors recommend a randomized trial to be performed.

A study of these reviews may suggest a bias against the single-layer closure with a greater chance of rupture in a future trial of labor; however, such a conclusion may be simplistic due to the fact that there is at present no conclusive evidence to prove that a double-layer closure would not result in rupture in a similar situation. The variables that exist in each case including previous vaginal delivery, birth weight, the integrity of the previous scar, a preexisting medical condition, and others make it well nigh impossible to dictate any superior methodology to suture a uterine incision at cesarean section. The debate rages on. However, suturing a thick muscular organ would at times necessitate a double-layer closure if at all only for achieving hemostasis. Flexibility of approach I suppose is the one rule we may rigidly follow.

\section{References}

1. Bujold E, Goyet M, Marcoux S, et al. The role of uterine closure in the risk of uterine rupture. Obstet Gynecol. 2010;116(1):43-50.

2. Roberge S, Chaiilet N, Boutin A, et al. Single- versus double-layer closure of the hysterotomy incision during cesarean delivery and risk of uterine rupture. Int J Gynecol Obstet. 2011;115(1):5-10.

3. Dodd J, Anderson E, Gates S, et al. Surgical techniques for uterine incision and uterine closure at the time of caesarean section. Cochrane Database of Syst Rev. 2008;. doi:10.1002/ 14651858.CD00472.pub2.

4. Gyamfi C, Gabor J, Gyamfi P, et al. Single- versus double-layer uterine incision closure and uterine rupture. $\mathrm{J}$ Mater. 2006;19(10):639-43.

5. Hudic I, Bujold E, Fatusic Z, et al. Risk of uterine rupture following locked vs unlocked single-layer closure. Med Arh. 2012;66(6):412-4. 